PROCEEDINGS OF THE

AMERICAN MATHEMATICAL SOCIETY

Volume 132, Number 6, Pages 1649-1658

S 0002-9939(04)07087-X

Article electronically published on January 27, 2004

\title{
NEW TRANSFORMATIONS FOR PAINLEVÉ'S THIRD TRANSCENDENT
}

\author{
N. S. WITTE
}

(Communicated by Mark J. Ablowitz)

\begin{abstract}
We present transformations relating the third transcendent of Painlevé with parameter sets located at the corners of the Weyl chamber for the symmetry group of the system, the affine Weyl group of the root system $B_{2}^{(1)}$, to those at the origin. This transformation entails a scaling of the independent variable, and implies additive identities for the canonical Hamiltonians and product identities for the $\tau$-functions with these parameter sets.
\end{abstract}

A curious anomaly has existed in the theory of the Painlevé transcendents for many years, for almost a century in fact. This anomaly is the existence of a particular transformation for the second Painlevé transcendent $q(t ; \alpha+1 / 2)$ that had no known analogue for any of the other transcendents. One recalls that the second Painlevé transcendent $q=q(t ; \alpha+1 / 2)$ is by definition a solution of the $\mathrm{P}_{\mathrm{II}}$ differential equation

$$
\frac{d^{2} q}{d t^{2}}=2 q^{3}+t q+\alpha
$$

with parameter $\alpha \in \mathbb{C}$. The transformation, found by Gambier in 1909 [5], relates solutions of the $\mathrm{P}_{\mathrm{II}}$ transcendent with parameter values at $v_{1}:=\alpha+1 / 2=0$ and $v_{1}=1 / 2$ (also between the solutions at $v_{1}=1 / 2$ and $v_{1}=1$, but this can be constructed from the previous one and the Bäcklund transformation $v_{1} \mapsto v_{1}+1$ ). The Bäcklund symmetries of $\mathrm{P}_{\mathrm{II}}$ are a realisation of the affine Weyl group associated with the root system $A_{1}^{(1)}$, and using

$$
\begin{aligned}
& v_{1} \mapsto v_{1}+1, \quad \text { shift, } \\
& v_{1} \mapsto-v_{1}, \quad \text { reflection, }
\end{aligned}
$$

transformations of this group, solutions for arbitrary $v_{1}$ can be related to a solution whose parameter $v_{1} \in(0,1 / 2]$ (or in $[1 / 2,1)$ ). This interval defines the Weyl chamber and the above transformation in question relates the solutions at the endpoints of this interval (see Figure 1).

Specifically the transformation takes the form

$$
\begin{aligned}
T & =-2^{-1 / 3} t, \\
2^{1 / 3} Q^{2}(T) & =\frac{d q(t)}{d t}+q^{2}(t)+1 / 2 t,
\end{aligned}
$$

Received by the editors January 26, 2002 and, in revised form, June 1, 2002.

2000 Mathematics Subject Classification. Primary 34M55, 33E17; Secondary 20F55.

Key words and phrases. Painlevé equations, Bäcklund transformations.

(C)2004 American Mathematical Society 
where $q(t):=q(t ; 0), Q(T):=q(T ; 1 / 2)$ and its inverse is

$$
\begin{aligned}
t & =-2^{1 / 3} T, \\
q(t) & =-2^{-1 / 3} \frac{1}{Q(T)} \frac{d Q(T)}{d T} .
\end{aligned}
$$

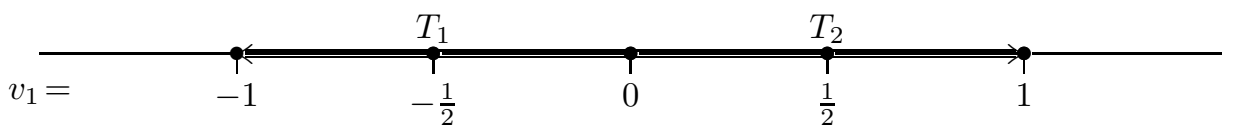

Figure 1. Parameter space $\left(v_{1}\right)$ associated with the simple roots of the root system $A_{1}^{(1)}$

We draw the reader's attention to some pertinent observations that distinguish this transformation from the Bäcklund (or equivalently Schlesinger) transformations (2). Firstly, the shift in the parameters is always the smallest possible shift, one half unit, on the root lattice whereas the Bäcklund transformation shift is twice this; secondly, the independent variable undergoes a scale change in contrast to remaining a fixed variable; thirdly, the transformation is quadratic in $q$ and $Q$ but linear in the derivatives. The purpose of this note is to demonstrate that such a transformation exists for $\mathrm{P}_{\mathrm{III}^{\prime}}$ as well, and to illustrate the many analogous properties that the new transformation for $\mathrm{P}_{\mathrm{III}^{\prime}}$ has with those of the $\mathrm{P}_{\mathrm{II}}$ transformation. After recognising these similarities one may well talk of a class of such transformations for all systems, $\mathrm{P}_{\mathrm{II}}$ to $\mathrm{P}_{\mathrm{VI}}$. Actually, an analogue of this class of transformations was noted by Okamoto 20 in the case of the fourth transcendent, but appeared not to be appreciated as such. Aside from the intrinsic interest of these transformations there are some practical motivations from the theory of random matrices where they arise as multiplicative identities for the probabilities that certain spectral intervals are free of eigenvalues [24.

We first present the context for these transformations by reviewing the understanding of the Bäcklund symmetries of $\mathrm{P}_{\mathrm{III}}$ and the underlying affine Weyl group structure. The $\mathrm{P}_{\mathrm{III}^{\prime}}$ differential equation for $q(t)$, in the convention of Okamoto [21], is

$$
\frac{d^{2} q}{d t^{2}}=\frac{1}{q}\left(\frac{d q}{d t}\right)^{2}-\frac{1}{t} \frac{d q}{d t}+\frac{q^{2}}{4 t^{2}}(\gamma q+\alpha)+\frac{\beta}{4 t}+\frac{\delta}{4 q},
$$

with the parameter identifications

$$
\alpha=-4 \eta_{\infty} v_{2}, \quad \beta=4 \eta_{0}\left(v_{1}+1\right), \quad \gamma=4 \eta_{\infty}^{2}, \quad \delta=-4 \eta_{0}^{2} .
$$

Here $\eta_{\infty}, \eta_{0}$ are fixed at unity, and all discussions of parameters will be in terms of the coordinates $\mathbf{v}=\left(v_{1}, v_{2}\right) \in \mathbb{C}^{2}$ on the root lattice. There is a Hamiltonian structure underlying the $\mathrm{P}_{\mathrm{III}}$ system $\{q, p ; t, H\}$ and the Hamiltonian is conventionally taken to be

$$
t H=q^{2} p^{2}-\left(\eta_{\infty} q^{2}+v_{1} q-\eta_{0} t\right) p+{ }_{1 / 2} \eta_{\infty}\left(v_{1}+v_{2}\right) q .
$$


The Hamilton equations of motions are then

$$
\begin{aligned}
& t q^{\prime}=2 q^{2} p-\eta_{\infty} q^{2}-v_{1} q+\eta_{0} t, \\
& t p^{\prime}=-2 q p^{2}+\left(2 \eta_{\infty} q+v_{1}\right) p-1 / 2 \eta_{\infty}\left(v_{1}+v_{2}\right),
\end{aligned}
$$

and $q(t)$ satisfies (5). Okamoto [21] and Kajiwara et al. [14] have identified two Bäcklund or Schlesinger transformations with the action on the parameters:

$$
T_{1} \cdot \mathbf{v}=\left(v_{1}+1, v_{2}+1\right), \quad T_{2} \cdot \mathbf{v}=\left(v_{1}+1, v_{2}-1\right) .
$$

The operators $T_{1}$ and $T_{2}$ can be constructed out of the generators $s_{0}, s_{1}, s_{2}$, which are reflections associated with the underlying $B_{2}$ root lattice, whose action (following [21] and [14]) on $\mathbf{v}, q, p$ is given in Table [1. According to Table 1] we have

$$
T_{1}=s_{0} s_{2} s_{1} s_{2}, \quad T_{2}=s_{2} s_{0} s_{2} s_{1} .
$$

See Figure 2 for the $B_{2}$ root lattice.

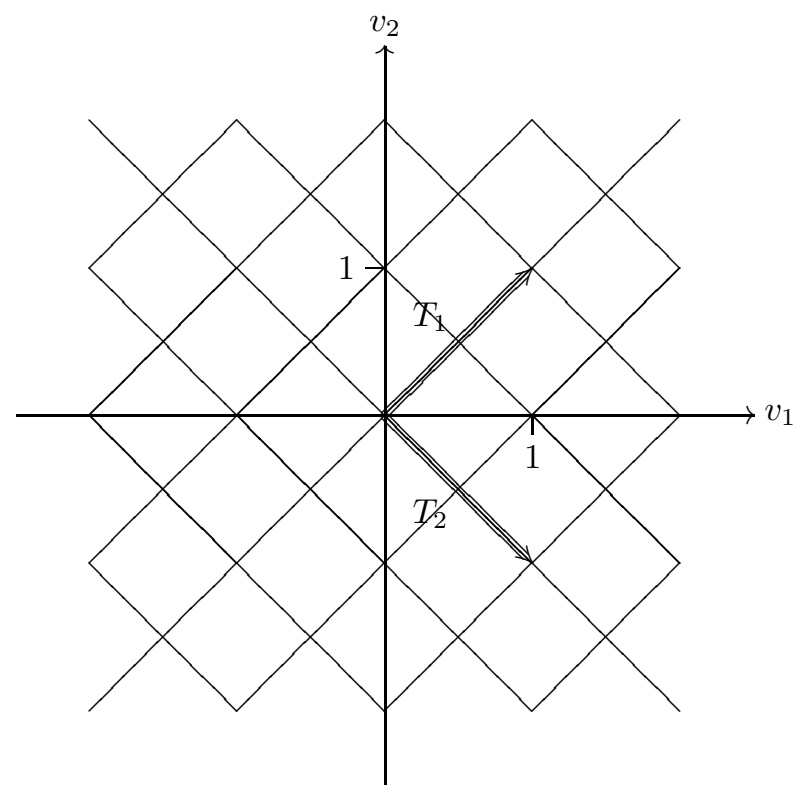

Figure 2. Parameter space $\left(v_{1}, v_{2}\right)$ associated with the root system $B_{2}^{(1)}$

Associated with the Hamiltonian is the $\tau$-function defined by

$$
H(t):=\frac{d}{d t} \log \tau \text {. }
$$

It has the property of being entire in $t \in \mathbb{C} \backslash\{0, \infty\}$ and having only simple zeros. One-parameter solutions, a particular type of classical solution, of $\mathrm{P}_{\mathrm{III}^{\prime}}$ are possible only when the parameters satisfy ([21], [18, 12])

$$
v_{2} \pm v_{1} \in 2 \mathbb{Z} \text {. }
$$




\begin{tabular}{|c||c|c|c|c|c|}
\hline & $v_{1}$ & $v_{2}$ & $p$ & $q$ & $t$ \\
\hline$s_{0}$ & $-1-v_{2}$ & $-1-v_{1}$ & $\frac{q}{t}\left[q(p-1)-\frac{1}{2}\left(v_{1}-v_{2}\right)\right]+1$ & $-\frac{t}{q}$ & $t$ \\
$s_{1}$ & $v_{2}$ & $v_{1}$ & $p$ & $q+\frac{v_{2}-v_{1}}{2(p-1)}$ & $t$ \\
$s_{2}$ & $v_{1}$ & $-v_{2}$ & $1-p$ & $-q$ & $-t$ \\
\hline
\end{tabular}

TABLE 1. Generators of the Bäcklund transformations realising a representation of the extended affine Weyl group for the root system $B_{2}^{(1)}$

The corresponding $\tau$-functions have the explicit Toeplitz determinant form involving Bessel functions,

$$
\operatorname{det}\left[I_{\nu+j-k}(\sqrt{t})+c K_{\nu+j-k}(\sqrt{t})\right]_{0 \leq j, k \leq n},
$$

$c, \nu \in \mathbb{C}$ or

$$
\operatorname{det}\left[J_{\nu+j-k}(\sqrt{t})+c Y_{\nu+j-k}(\sqrt{t})\right]_{0 \leq j, k \leq n} .
$$

Rational, or zero-parameter, solutions are possible only when ([12])

$$
v_{2} \pm v_{1} \in 2 \mathbb{Z}+1
$$

These are constructed by recurrences derived using the operators (11) from a seed solution. Additional studies of the Bäcklund transformations and exact solutions of the third Painlevé equation from various perspectives are given in [15], [8], [9], [10], 1], [2], 17, [16], 23], 7], 6].

Let us now return to Gambier's transformation (3), (4). Expressed in canonical variables, (3) takes the form

$$
\begin{aligned}
& T=-2^{-1 / 3} t, \\
& Q=2^{-1 / 6} \sqrt{p}, \\
& P=-2^{-1 / 3}\left[1 /{ }_{2} t-p+q \sqrt{2 p}\right],
\end{aligned}
$$

and the inverse transformation (4) is

$$
\begin{aligned}
& t=-2^{1 / 3} T, \\
& q=-2^{-1 / 3} \frac{P-Q^{2}-T / 2}{Q}, \\
& p=2^{1 / 3} Q^{2} .
\end{aligned}
$$

The corresponding transformation for $\mathrm{P}_{\mathrm{III}^{\prime}}$ is given in the following result.

Proposition 1. The canonical variables of the $\mathrm{P}_{\text {III' }^{\prime}}$ Hamiltonian system $\{q, p ; t, h\}$ with $\mathbf{v}=(0,0)$ are related to the $\mathrm{P}_{\mathrm{III}^{\prime}}$ Hamiltonian system $\{Q, P ; T, H\}$ with $\mathbf{v}=$ 
$(-1,0)$ by the direct canonical transformation

$$
\begin{aligned}
& T=1 / 4 t \\
& Q=i \frac{\sqrt{t}}{2}(\sqrt{p}+\sqrt{p-1}), \\
& P=\frac{i}{\sqrt{t}} \frac{1 / 2+q \sqrt{p} \sqrt{p-1}-i \sqrt{t} \sqrt{p}}{\sqrt{p}+\sqrt{p-1}} .
\end{aligned}
$$

The inverse of this transformation is

$$
\begin{aligned}
& t=4 T \\
& q=-2 T Q \frac{2 Q^{2}-4 Q^{2} P-Q-2 T}{\left(Q^{2}+T\right)\left(Q^{2}-T\right)}, \\
& p=-\frac{\left(Q^{2}-T\right)^{2}}{4 T Q^{2}} .
\end{aligned}
$$

First Proof. One can directly verify that $Q(T)$ satisfies the differential equation (5) with appropriate parameters by hand or otherwise.

Second Proof. One can adapt Gromak's method for $\mathrm{P}_{\mathrm{II}}[12$ to this case in the following sequence of steps. In the first step one eliminates $q(t)$ instead of $p(t)$ from Hamilton's equations of motion (91) and arrives at

(21) $p^{\prime \prime}=1 / 2\left(\frac{1}{p}+\frac{1}{p-1}\right) p^{\prime 2}-\frac{p^{\prime}}{t}+\frac{2}{t} p(1-p)+\frac{1}{4 t^{2}} \frac{\left(v_{1}+v_{2}\right)^{2}(2 p-1)-4 v_{1} v_{2} p^{2}}{2 p(1-p)}$.

Employing the transformation

$$
p=\frac{y}{y-1},
$$

one finds that $y(t)$ satisfies the standard form for Painlevé's fifth transcendent with the parameters

$$
\alpha=1 / 8\left(v_{1}-v_{2}\right)^{2}, \quad \beta=-1 / 8\left(v_{1}+v_{2}\right)^{2}, \quad \gamma=2, \quad \delta=0,
$$

and in this sense this particular form of $\mathrm{P}_{\mathrm{V}}$ is dual to the general $\mathrm{P}_{\mathrm{III}}$ ([13], [12], [11, [9]). In the second step we need to transform this into a $\mathrm{P}_{\mathrm{III}}$ transcendent, but the crucial point is that one should not use a contact transformation to effect this under the most general conditions but employ a point transformation which will only work for particular parameters. By setting $v_{1}=v_{2}=0$, such a transformation is

$$
y=\left(\frac{u+1}{u-1}\right)^{2}
$$

which can be applied to $\mathrm{P}_{\mathrm{V}}$ when $\alpha=\beta=0$ and general $\gamma, \delta$ [22]. The resulting differential equation for $u(t)$ is that of a $\mathrm{P}_{\mathrm{III}}$ transcendent with $\alpha=-1 / 2, \beta=$ $1 / 2, \gamma=\delta=0$, which is in a nonstandard form. One can convert this to a standard form of $\mathrm{P}_{\text {III }}$ with the transformation

$$
\begin{gathered}
t=z^{2}, \\
u(t)=v^{2}(z),
\end{gathered}
$$


and the parameters become $\alpha=0, \beta=0, \gamma=-1, \delta=1$. In the next step one requires that the $\mathrm{P}_{\mathrm{III}}$ system be transformed into our original $\mathrm{P}_{\text {III' }}$ system with the transformation

$$
\begin{aligned}
x & =z^{2}, \\
w(x) & =z v(z),
\end{aligned}
$$

and this does not alter the parameters. In the final step one has to restore the original scaling of the $\mathrm{P}_{\mathrm{III}}$ system with $\gamma=4, \delta=-4$, and all that is necessary is a scaling of the independent variable $T=1 /{ }_{4} x=1 / 4 t$. The other parameters $\alpha, \beta$ are unchanged, and so we have our original system but with $v_{1}=-1, v_{2}=0$. The composition of all these transformations yields the stated result.

Third Proof. The inverse transformation can be written as

$$
\begin{aligned}
t & =4 T, \\
q(t) & =-2 T Q(T) \frac{Q(T)-2 T Q^{\prime}(T)}{\left(Q^{2}(T)+T\right)\left(Q^{2}(T)-T\right)},
\end{aligned}
$$

and this can be recognised as a degenerative form of the Riccati transformations

$$
\begin{aligned}
z & =\mu t, \\
u(z) & =\frac{v^{\prime}(t)^{2}+A_{2}(v) v^{\prime}(t)+A_{4}(v)}{B_{2}(v) v^{\prime}(t)+B_{4}(v)},
\end{aligned}
$$

first proposed by Fokas and Ablowitz [3], but generalised to include a scale change in the independent variable. Here $A_{2}(v), B_{2}(v)$ are quadratic polynomials of $v(t)$ with $t$-dependent coefficients and $A_{4}(v), B_{4}(v)$ are quartic polynomials, and in this particular form the $v^{\prime 2}$ and $B_{2}(v) v^{\prime}$ terms are absent. One can derive (27), as well as the Gambier transformation for $\mathrm{P}_{\mathrm{II}}$, using the approach of [3].

Remark 1. The appearance of branch points at $p=0,1$ in the transformation (19) is a consequence of the fixed singularities at these points in the differential equation (21) that is dual to the general $\mathrm{P}_{\mathrm{III}}$ equation. Similarly the transformation for $\mathrm{P}_{\mathrm{II}}$ (17) has a branch point at $p=0$ which corresponds to the singularity in the differential equation for PXIV. The square-root nature of the branches naturally appears from the inversion of the quadratic rational transformation given by the last member of (20).

Remark 2. The solution of the $\mathrm{P}_{\mathrm{III}^{\prime}}$ differential equation $q(t)$ with parameters $(0,0)$ is related to another solution $Q(T)$ of this equation with parameters $(-1,0)$ by the direct transformation

$$
\begin{aligned}
T & =1 / 4 t, \\
Q(T) & =i \frac{\sqrt{t}}{2 q(t)}\left(\sqrt{\frac{t q^{\prime}(t)-q^{2}(t)-t}{2}}+\sqrt{\frac{t q^{\prime}(t)+q^{2}(t)-t}{2}}\right) .
\end{aligned}
$$

This follows by eliminating $p$ from (19) using the Hamilton equations (9).

Remark 3. Using the relations (26) one can recast the transformations between the origin and $(-1,0)$ for the $\mathrm{P}_{\mathrm{III}^{\prime}}$ system into equivalent transformations for the $\mathrm{P}_{\mathrm{III}}$ system. If one employs a notation for the $\mathrm{P}_{\mathrm{III}}$ transcendent $\mu(s)$ with parameters $\alpha=0, \beta=4, \gamma=4, \delta=-4$ and $M(S)$ with parameters $\alpha=0, \beta=0, \gamma=$ 
$4, \delta=-4$, then one has the direct and inverse transformations

$$
\begin{aligned}
& S=1 / 2 s, \\
& M(S)=\frac{i}{2 \sqrt{s} \mu(s)}\left[\sqrt{\mu(s)+s \mu^{\prime}(s)+2 s\left(\mu^{2}(s)-1\right)}\right. \\
& \left.+\sqrt{\mu(s)+s \mu^{\prime}(s)-2 s\left(\mu^{2}(s)+1\right)}\right], \\
& \mu(s)=\frac{M(S) M^{\prime}(S)}{\left(M^{2}(S)+1\right)\left(M^{2}(S)-1\right)} .
\end{aligned}
$$

Remark 4. One might think that transformations from the origin to other nearest neighbours along the axes in parameter space (recall Figure 2) would be simply expressible. One can indeed find these by composing Bäcklund transformations with the above transformation, and one has the following corollary. In the following discussion we employ a renaming of the variables $Q, P, H \mapsto Q_{W}, P_{W}, H_{W}$ and a notation using the four primary compass directions $S, E, N, W$.

Corollary 2. The canonical variables of the $\mathrm{P}_{\text {III' }}$ Hamiltonian system $\{q, p ; t, h\}$ with $\mathbf{v}=(0,0)$ are related to the $\mathrm{P}_{\mathrm{III}}$ Hamiltonian system $\left\{Q_{S}, P_{S} ; T, H_{S}\right\}$ with $\mathbf{v}=(0,-1)$ by the direct canonical transformation with $T=1 / 4$ t and

$$
\begin{aligned}
Q_{S} & =i \frac{\sqrt{t}}{2} \frac{1}{\sqrt{p}+\sqrt{p-1}} \frac{1 / 2+q \sqrt{p} \sqrt{p-1}-i \sqrt{t} \sqrt{p-1}}{1 / 2-q \sqrt{p} \sqrt{p-1}+i \sqrt{t} \sqrt{p-1}}, \\
P_{S} & =-\frac{i}{\sqrt{t}}(\sqrt{p}+\sqrt{p-1})[1 / 2-q \sqrt{p} \sqrt{p-1}+i \sqrt{t} \sqrt{p}],
\end{aligned}
$$

and are related to the $\mathrm{P}_{\mathrm{III}^{\prime}}$ Hamiltonian system $\left\{Q_{E}, P_{E} ; T, H_{E}\right\}$ with $\mathbf{v}=(1,0)$ by the direct canonical transformation

$$
\begin{aligned}
& Q_{E}=-i \frac{\sqrt{t}}{2}(\sqrt{p}+\sqrt{p-1})\left\{1-\frac{1}{1 / 2+q \sqrt{p} \sqrt{p-1}-i \sqrt{t} \sqrt{p-1}}\right.\left.-\frac{1}{1 / 2+q \sqrt{p} \sqrt{p-1}+i \sqrt{t} \sqrt{p}}\right\}, \\
& P_{E}=-\frac{i}{\sqrt{t}} \frac{1 / 2+q \sqrt{p} \sqrt{p-1}+i \sqrt{t} \sqrt{p}}{\sqrt{p}+\sqrt{p-1}},
\end{aligned}
$$

and to the $\mathrm{P}_{\mathrm{III}}$ Hamiltonian system $\left\{Q_{N}, P_{N} ; T, H_{N}\right\}$ with $\mathbf{v}=(0,1)$ by the direct canonical transformation

$$
\begin{aligned}
Q_{N} & =-i \frac{\sqrt{t}}{2} \frac{1}{\sqrt{p}+\sqrt{p-1}} \frac{1 / 2+q \sqrt{p} \sqrt{p-1}+i \sqrt{t} \sqrt{p}}{1 / 2-q \sqrt{p} \sqrt{p-1}-i \sqrt{t} \sqrt{p}} \\
P_{N} & =\frac{i}{\sqrt{t}} \frac{1 / 2-q \sqrt{p} \sqrt{p-1}-i \sqrt{t} \sqrt{p}}{\sqrt{p}-\sqrt{p-1}} .
\end{aligned}
$$

Proof. These are found by composing the transformation (19) with the Bäcklund transformations (11).

These transformations attain a clearer form when one considers the relationship between the Hamiltonians defined by these canonical transformations. To illustrate this we consider the case of $\mathrm{P}_{\mathrm{II}}$ first. Denoting $H_{0}:=H_{\mathrm{II}}\left(q, p ; t ; v_{1}=0\right)$ and $H_{1 / 2}:=$ 
$H_{\mathrm{II}}\left(Q, P ; T, v_{1}=1 / 2\right)$, we find that by employing the transformations (3) directly, or by constructing the generating function for the canonical transformation, that

$$
\begin{aligned}
H_{0} & =-2^{-1 / 3}\left[P^{2}-2\left(Q^{2}+T / 2\right) P+T^{2} / 4\right] \\
& =-2^{-1 / 3}\left[2 H_{1 / 2}+Q+T^{2} / 4\right] .
\end{aligned}
$$

But we note that $H_{-1 / 2}:=H_{\mathrm{II}}\left(T, v_{1}=-1 / 2\right)$ is given by the Bäcklund transformation

$$
H_{-1 / 2}=T_{1} H_{1 / 2}=H_{1 / 2}+Q,
$$

so that we have the additive relation

$$
H_{0}+t^{2} / 8=-2^{-1 / 3}\left(H_{1 / 2}+H_{-1 / 2}\right),
$$

and a multiplicative relation for the corresponding $\tau$-functions

$$
\exp \left(t^{3} / 24\right) \tau_{0}(t)=\tau_{-1 / 2}(T) \tau_{1 / 2}(T) .
$$

If one takes the definition of the $\phi$-factors or $\tau$-cocycles $[19$,

$$
\tau(t):=\exp \left(-t^{3} / 48\right) \phi(t),
$$

then the multiplicative relation is

$$
\phi_{0}(t)=\phi_{-1 / 2}(T) \phi_{1 / 2}(T) .
$$

The above identity has arisen in random matrix theory as a result of reconciling two different calculations [4] for the cumulative distribution of the largest eigenvalue of a random Hermitian matrix drawn from the Gaussian unitary ensemble.

We also have analogous relations in the case of $\mathrm{P}_{\mathrm{III}^{\prime}}$.

Proposition 3. The Hamiltonians for the parameter sets $(-1,0),(0,-1),(1,0)$, $(0,1)$ are related to the Hamiltonian at the origin by the additive relation

$$
T H_{W}(T)+T H_{S}(T)+T H_{E}(T)+T H_{N}(T)=t h(t)-1 / 4 .
$$

The canonical momenta are related in a similar way,

$$
P_{W}+P_{S}+P_{E}+P_{N}=4 p .
$$

The $\tau$-functions are related in the multiplicative form

$$
T^{1 / 4} \tau_{W}(T) \tau_{S}(T) \tau_{E}(T) \tau_{N}(T)=\tau(t) .
$$

Proof. By employing the canonical transformations (19), (33), (34), (35) we find that the Hamiltonians at the four corners $(-1,0),(0,-1),(1,0),(0,1)$ are related to the Hamiltonian at the origin by

$$
\begin{aligned}
& T H_{W}(T)=1 / 4 \operatorname{th}(t)-1 / 16+1 / 4[-1 / 2-q \sqrt{p} \sqrt{p-1}+i \sqrt{t}(\sqrt{p}-\sqrt{p-1})] \\
& T H_{S}(T)=1 / 4 \operatorname{th}(t)-1 / 16+1 / 4[1 / 2+q \sqrt{p} \sqrt{p-1}-i \sqrt{t}(\sqrt{p}+\sqrt{p-1})] \\
& T H_{E}(T)=1 / 4 \operatorname{th}(t)-1 / 16+1 / 4[-1 / 2-q \sqrt{p} \sqrt{p-1}-i \sqrt{t}(\sqrt{p}-\sqrt{p-1})], \\
& T H_{N}(T)=1 / 4 \operatorname{th}(t)-1 / 16+1 / 4[1 / 2+q \sqrt{p} \sqrt{p-1}+i \sqrt{t}(\sqrt{p}+\sqrt{p-1})],
\end{aligned}
$$

and their sum yields (42). Utilising the second members of (19), (33), (34), (35), relation (43) follows directly. The final product relation for the $\tau$-functions is the result of integrating (42). 


\section{ACKNOWLEDGMENTS}

It is a pleasure to acknowledge the many and wide-ranging discussions with Peter Forrester, the valuable advice and insight of Chris Cosgrove and the observations made by Nalini Joshi and Peter Clarkson. This research has been supported by the Australian Research Council.

\section{REFERENCES}

1. H. Airault, Rational solutions of Painlevé equations, Stud. Appl. Math. 61 (1979), 31-53. MR 80e: 58005

2. L. A. Bordag and A. V. Kitaev, Transformations of the solutions of the third and of the fifth Painlevé equations and its partial solutions, Joint Inst. Nuclear Res., Dubna, 1985, R5-85-740. MR 88h:34004

3. A. S. Fokas and M. J. Ablowitz, On a unified approach to transformations and elementary solutions of Painlevé equations, J. Math. Phys. 23 (1982), 2033-2042. MR 84b:34004

4. P. J. Forrester and N. S. Witte, Application of the $\tau$-function theory of Painlevé equations to random matrices: PIV, PII and the GUE, Commun. Math. Phys. 219 (2001), 357-398. MR 2003a:82031

5. B. Gambier, Sur les équations différentielles du second ordre et du premier degré dont l'intégrale générale est a points critiques fixes, Acta Math. 33 (1909), 1-55.

6. P. R. Gordoa, N. Joshi, and A. Pickering, Mappings preserving locations of movable poles. II. The third and fifth Painlevé equations, Nonlinearity 14 (2001), no. 3, 567-582. MR 2002b:34134

7. P. R. Gordoa, N. Joshi, and A. Pickering, Truncation-type methods and Bäcklund transformations for ordinary differential equations: the third and fifth Painleve equations, Glasg. Math. J. 43A (2001), 23-32, Integrable systems: linear and nonlinear dynamics (Islay, 1999). MR 2002h:34189

8. V. I. Gromak, The solutions of Painlevé's third equation, Differencial'nye Uravnenija 9 (1973), 2082-2083, 2118; translation in Differ. Equ. 9 (1973), 1599-1600. MR 49:5434

9. V. I. Gromak, On the theory of Painlevé's equations, Differencial'nye Uravnenija 11 (1975), 373-376, 398; translation in Differ. Equ. 11 (1975), 285-287. MR 51:13321

10. V. I. Gromak, The solutions of Painlevé's fifth equation, Differencial'nye Uravnenija 12 (1976), 740-742, 775; translation in Differ. Equ. 12 (1976), 519-521. MR 55:3377

11. V. I. Gromak, Reducibility of the Painlevé equations, Differencial'nye Uravnenija 20 (1984), 1674-1683; translation in Differ. Equ. 20 (1984), 1191-1198. MR 86a:34015]

12. V. I. Gromak, Bäcklund transformations of Painlevé equations and their applications, The Painlevé Property: One Century later (R. Conte, ed.), CRM Series in Mathematical Physics, Springer-Verlag, New York, 1999, pp. 687-734. MR 2000m:34011

13. V. I. Gromak and G. V. Filipuk, On functional relations between solutions of the fifth Painlevé equation, Differencial'nye Uravnenija 37 (2001), 586-591, 717; translation in Differ. Equ. 37 (2001), 614-620. MR 2002f:34219

14. K. Kajiwara, T. Masuda, M. Noumi, Y. Ohta, and Y. Yamada, Determinant formulas for the Toda and discrete Toda equations, Funkcialaj Ekvacioj 44 (2001), 291-307, solv-int/9908007. MR 2002h:37140

15. N. A. Lukaševič, On the theory of Painlevé's third equation, Differencial'nye Uravnenija 3 (1967), 1913-1923; translation in Differ. Equ. 3 (1967), 994-999. MR 37:5451

16. E. L. Mansfield and H. N. Webster, On one-parameter families of Painlevé III, Studies Appl. Math. 101 (1998), 321-341. MR 99g:34025

17. A. E. Milne, P. A. Clarkson, and A. P. Bassom, Bäcklund transformations and solution hierarchies for the third Painlevé equation, Stud. Appl. Math. 98 (1997), no. 2, 139-194. MR 98b:34023

18. Y. Murata, Classical solutions of the third Painlevé equation, Nagoya Math. J. 139 (1995), 37-65. MR 96h:34016

19. M. Noumi, Painlevé equations: An introduction from the symmetric point of view, Asakura Shoten Publishing, Tokyo, 2000, in Japanese.

20. K. Okamoto, Studies on the Painlevé equations. III. Second and fourth Painlevé equations, $P_{\mathrm{II}}$ and $P_{\mathrm{IV}}$, Math. Ann. 275 (1986), no. 2, 221-255. MR 88m:58064 
21. K. Okamoto, Studies on the Painlevé equations. IV. Third Painlevé equation $P_{\mathrm{III}}$, Funkcial. Ekvac. 30 (1987), no. 2-3, 305-332. MR 88m:58065

22. P. Painlevé, Sur les équations différentielles du second ordre et d'ordre supérieur dont l'intégrale générale est uniforme, Acta Math. 25 (1902), 1-85.

23. H. Umemura and H. Watanabe, Solutions of the third Painlevé equation. I, Nagoya Math. J. 151 (1998), 1-24. MR 99k:34016

24. N. S. Witte, Gap probabilities for double intervals in Hermitian random matrix ensembles as $\tau$-functions - the Bessel kernel case, in preparation, 2001.

Department of Mathematics and Statistics, University of Melbourne, Victoria 3010 , Australia

E-mail address: N.Witte@ms.unimelb.edu.au 